\title{
Increased Levels of Asymmetric Dimethylarginine (ADMA) in Patients with Ankylosing Spondylitis
}

\author{
Ismail Sari ${ }^{1}$, Levent Kebapcilar ${ }^{1}$, Ahmet Alacacioglu ${ }^{1}$, Oktay Bilgir ${ }^{1}$, Yasar Yildiz ${ }^{1}$, \\ Ali Taylan ${ }^{2}$, Arif Yuksel ${ }^{1}$ and Didem L. Kozaci ${ }^{3}$
}

\begin{abstract}
Objective Endothelial dysfunction is present in ankylosing spondylitis (AS). However, the etiology of events is still unclear. The aim of the present study was to investigate whether there are abnormalities in nitric oxide (NO) metabolism and endothelin-1 (ET-1) in AS patients.

Methods Subjects without any classical cardiovascular (CV) risk factors were studied. Fasting glucose, serum lipids, high sensitive CRP (hsCRP), ESR, asymmetric dimethylarginine (ADMA) and ET-1 were studied. Patients were also evaluated with the Bath Ankylosing Spondylitis Metrology Index, Bath Ankylosing Spondylitis Functional Index, and the Bath Ankylosing Spondylitis Disease Activity Index.

Results A total of 48 AS patients $(38.6 \pm 10.6$ years; $36 \mathrm{M} / 12 \mathrm{~F})$ and 38 controls $(36.4 \pm 11.1$ years; $27 \mathrm{M} / 11 \mathrm{~F})$ were studied. Acute phase reactants including hsCRP, and ESR were significantly increased in the patients group $(\mathrm{p}<0.05)$. Serum ADMA concentrations were also significantly higher in AS than in controls. Plasma levels of ET-1 did not differ between the groups $(\mathrm{p}>0.05)$. Comparison of three groups (conventional and anti-TNF treatment groups and controls) revealed that ADMA was significantly higher in the conventional treated AS than in controls. The levels of ADMA were not different between anti-TNF group and healthy subjects. Plasma ET-1 concentrations were similar between groups $(\mathrm{p}>0.05)$. Correlation analysis yielded significant correlations between ADMA, hsCRP, LDL cholesterol, HDL cholesterol and triglycerides $(\mathrm{p}<0.05)$. Conclusion The increased ADMA levels obtained in a group of relatively young AS patients who did not have classical CV risk factors suggest that NO metabolism is impaired in AS. On the other hand, anti-TNF treatments may have a beneficial effect on vascular function in AS.
\end{abstract}

Key words: asymmetric dimethylarginine, endothelin-1, endothelium, ankylosing spondylitis, atherosclerosis

(Inter Med 48: 1363-1368, 2009)

(DOI: 10.2169/internalmedicine.48.2193)

\section{Introduction}

Ankylosing spondylitis (AS) is a chronic inflammatory disease of the spine and sacroiliac joints of unknown etiology (1). AS may affect extraarticular tissues such as eyes, digestive tract, heart, and kidneys (2). The severity of cardiac involvement varies greatly ranging from asymptomatic to severe considerable problems such as severe heart failure, and arrhythmias (3). Some studies revealed an increased prevalence of cardiovascular (CV) mortality in AS (4-8). Re- cently, an impaired endothelial function, by using ultrasonography, has been demonstrated in a relatively young age group of AS patients without known classical CV risk factors (9). However the etiology of events is still unclear. In this study, we aimed to determine the blood markers of asymmetric dimethylarginine (ADMA), a marker in nitric oxide (NO) metabolism, and endothelin-1 (ET-1) in AS patients, who were free of $\mathrm{CV}$ risk factors. We also investigated the effects of conventional and anti-tumor necrosis factor alpha (TNF- $\alpha$ ) treatments on ADMA and ET-1 levels.

\footnotetext{
${ }^{1}$ Department of Internal Medicine, Izmir Bozyaka Training and Research Hospital, İzmir, Turkey, ${ }^{2}$ Department of Internal Medicine, Izmir Tepecik Training and Research Hospital, İzmir, Turkey and ${ }^{3}$ Department of Biochemistry, Adnan Menderes University School of Medicine, Aydin, Turkey

Received for publication February 21, 2009; Accepted for publication April 29, 2009

Correspondence to Dr. Ismail Sari, ismailsari35@gmail.com
} 


\section{Materials and Methods}

\section{Patients and controls}

The sample size was calculated by using the results of previous studies that investigated the levels of ADMA (1012), and ET-1 (13-15) based on $\alpha=0.05$ and a power of $80 \%$. At least 13 patients (12 for endothelin, 13 for ADMA) were needed per group. A total of 60 AS patients, diagnosed according to the Modified New York criteria (16) (mean age $39.9 \pm 10.6$ years; 42 men $[\mathrm{M}], 18$ women $[\mathrm{F}])$, and 60 healthy control subjects (mean age $36.7 \pm 10.5$ years, $34 \mathrm{M} / 26$ F) were included in the study. Controls were recruited from the relatives of the health professionals and blood donors without any known chronic disease. Exclusion criteria were as follows: hypertension (HT), diabetes mellitus (DM), hyperlipidemia, and smoking. Patients who had been treated with systemic corticosteroids within 8 weeks before the study were also excluded. Disease duration, biological treatment usage and duration were recorded. Written informed consent was obtained from each subject, and research protocols were approved by the Ethical Committee of our institution. 12 AS patients (2 DM, 5 dyslipidemia and $5 \mathrm{HT}$ ) and 22 controls (3 DM, 14 dyslipidemia and 5 HT) were excluded from the study. Study was performed on the remaining 48 patients and 38 controls. This study was performed between March 2008 and October 2008.

\section{Definition of variables}

Hypertension: Average systolic BP $\geq 140 \mathrm{mmHg}$ or average diastolic $\mathrm{BP} \geq 90 \mathrm{mmHg}$, or receiving treatment for HT.

Dyslipidemia: Total cholesterol level $\geq 260 \mathrm{mg} / \mathrm{dL}$ or low density lipoprotein (LDL) cholesterol $\geq 160 \mathrm{mg} / \mathrm{dL}$ or the use of lipid lowering medication.

Diabetes: Current use of medications prescribed to treat DM or fasting serum glucose levels $\geq 126 \mathrm{mg} / \mathrm{dL}$.

Smoking: Any tobacco use in the past 30 days.

\section{Laboratory evaluation}

Following an overnight fasting, venous blood samples were collected for the measurement of laboratory tests in the morning (8:00-9:00 A.M). Erythrocyte sedimentation rate (ESR), fasting blood glucose, and serum lipids (total cholesterol, HDL cholesterol, LDL cholesterol and triglycerides) were studied during the same day. Serum and plasma samples for the measurement of high sensitive C-reactive protein (hsCRP), ADMA and ET-1 were preserved at $-80^{\circ} \mathrm{C}$ until analysis. Analyses of hsCRP, ADMA, and ET-1 were performed by using commercially available ELISA kits in accordance with the supplier's instructions. Other laboratory tests were measured according to standard procedures.

hsCRP: Serum hsCRP was determined using a commercially available sandwich ELISA kit (DRG International, USA, Cat No: EIA-3954). Diluted serum samples and a horseradish-peroxidase labelled secondary goat anti-human CRP antibody were applied to the ELISA plates coated with a mouse monoclonal anti-human CRP antibody according to the manufacturer's instructions. Color was developed with 3,3' 5' 5-tetramethylbenzidine and absorbance was measured at $450 \mathrm{~nm}$ against standard curves. Mean values were reported. The sensitivity of hsCRP was $0.1 \mathrm{mg} / \mathrm{L}$. The intraassay and inter-assay coefficient of variations were $2.3 \%$ and $2.5 \%$, respectively.

ADMA: Serum ADMA concentration was determined by using a competitive 96-well plate ADMA-ELISA kit (DLD, Diagnostika GMBH, Hamburg, Germany) following the supplier's protocol. This immunometric assay is based on the competition of acylated ADMA in samples with solid phase bound ADMA for a fixed number of rabbit anti-ADMA antiserum binding sites. ADMA shows negligible cross reactivity with L-arginine $(<0.02 \%)$ and other endogenous derivatives of L-arginine. Coefficients of variation are 8.3-10.3\%; and $4.5-7.5 \%$ for inter-assay and intra-assay, respectively. The ELISA assay can accurately measure ADMA concentrations over the full range of physiologically-relevant concentrations (i.e. $0.05 \mu \mathrm{mol} / \mathrm{L}$ to $2 \mu \mathrm{mol} / \mathrm{L}$ ). The specificity of ADMA ELISA kit is $100 \%$ and the sensitivity is $0.05 \mu \mathrm{mol} /$ L.

Endothelin -1: Plasma endothelin was determined by using $100 \mu \mathrm{L}$ of supernatant per well of a 96-well plate with a endothelin EIA kit (Cayman Chemical Company, Ann Arbor, MI, USA Cat No:583151) following the supplier's protocol. This immunometric assay is based on a doubleantibody 'sandwich' technique and permits endothelin measurements within the range of $0-250 \mathrm{pg} / \mathrm{mL}$, typically with a limit of detection of $1,5 \mathrm{pg} / \mathrm{mL}$. Monoclonal anti ET-1 antibody in this kit had a cross reactivity of $100 \%$ with ET-2 and $100 \%$ with ET-3. Samples were assessed with no prior purification. In this method monoclonal antibody specific to endothelin and acetylcholinesterase: Fab' Conjugate (AchE: Fab') bind to different epitopes on the Endothelin-1 molecule and forming sandwich. This sandwich is immobilized on the plate so the excess reagents are washed away. The concentration of analyte is detected by measuring the enzymatic activity of the AchE by adding Ellman's Reagent [5, 5'-dithiobis-(2-nitrobenzoic acid) or DTNB] which contains the substrate for AchE. The addition of Ellman's Reagent produces a yellow-colored product which can be measured spectrophotometrically. The intensity of the color is directly proportional to the amount of bound conjugate which in turn is proportional to the concentration of the endothelin. The intra- and inter-assay coefficients of variation of the method were 5 and $6 \%$, respectively.

\section{Other measurements}

Spinal mobility was assessed by the Bath Ankylosing Spondylitis Metrology Index (17). Patients were also evaluated with the Bath Ankylosing Spondylitis Functional Index (BASFI) (18) and the Bath Ankylosing Spondylitis Disease Activity Index (BASDAI) (19). BMI and waist circumfer- 
Table 1. Clinical and Laboratory Characteristics of AS Patients and Controls

\begin{tabular}{|c|c|c|c|}
\hline & AS patients $(n=48)$ & $\begin{array}{l}\text { Controls } \\
(n=38)\end{array}$ & $p$ value \\
\hline Age (yr) & $38.6 \pm 10.6$ & $36.4 \pm 11.1$ & 0.3 \\
\hline Sex (M/F) & $36 / 12$ & $27 / 11$ & 0.8 \\
\hline BMI $\left(\mathrm{kg} / \mathrm{m}^{2}\right)$ & $25.4 \pm 4.5$ & $25.2 \pm 3.7$ & 0.8 \\
\hline Waist circumference $(\mathrm{cm})$ & $85.7 \pm 11.4$ & $83.8 \pm 10.8$ & 0.4 \\
\hline Mean SBP $(\mathrm{mmHg})$ & $111 \pm 10.8$ & $110 \pm 11$ & 0.8 \\
\hline Mean DBP (mmHg) & $68 \pm 7$ & $69 \pm 6.6$ & 0.6 \\
\hline Fasting glucose (mg/dL) & $91.4 \pm 11.9$ & $92.3 \pm 11.3$ & 0.7 \\
\hline Total Cholesterol (mg/dL) & $175.5 \pm 32.9$ & $186.1 \pm 37.1$ & 0.2 \\
\hline HDL Cholesterol (mg/dL) & $46.6 \pm 11.1$ & $48.8 \pm 11.8$ & 0.4 \\
\hline LDL Cholesterol (mg/dL) & $109.2 \pm 29$ & $112.6 \pm 25.8$ & 0.6 \\
\hline Triglyceride (mg/dL) & $96.6 \pm 46$ & $106.8 \pm 59.1$ & 0.4 \\
\hline${ }^{\dagger} \mathrm{ESR}(\mathrm{mm} / \mathrm{h})$ & $11 \pm 19$ & $3 \pm 6$ & $<0.001$ \\
\hline hsCRP (mg/L) & $9 \pm 5.5$ & $4.2 \pm 3.6$ & $<0.001$ \\
\hline ADMA ( $\mu \mathrm{mol} / \mathrm{L})$ & $1.6 \pm 1$ & $0.9 \pm 0.9$ & 0.003 \\
\hline Endotelin (pg/mL) & $19 \pm 3.9$ & $20.4 \pm 5.3$ & 0.2 \\
\hline
\end{tabular}

ence were calculated.

\section{Statistical analysis}

The Kolmogorov-Smirnov test was used to determine if continuous variables were normally distributed. For normally distributed data, results were presented as mean \pm standard deviation (SD) and comparison between groups of continuous variables was performed by using the Student's $t$ test. When the variables were found not to be normally distributed (ESR, and BASMI), results were given as median \pm interquartile range (IQR) comparisons between groups of data were made using Mann-Whitney U test. Differences between categorical variables were analyzed by fisher's exact test. One-way ANOVA in conjunction with Tukey's HSD test or Kruskal-Wallis tests (ESR, and BASMI) were applied when comparing multiple groups. Relationships between variables were analyzed using Pearson or Spearman's rank correlation coefficients (ESR, and BASMI). The statistical analysis was carried out by using Statistical Package of Social Science (SPSS), version 13.0 (SPSS Inc., Chicago, IL). A $p$ value of $<0.05$ was considered as statistically significant.

\section{Results}

The study group consisted of 48 AS patients $(38.6 \pm 10.6$ years; $36 \mathrm{M} / 12 \mathrm{~F})$ and 38 healthy controls $(36.4 \pm 11.1$ years; $27 \mathrm{M} / 11 \mathrm{~F})$. There were no differences with respect to the age and sex distribution $(p>0.05)$. Anthropometrical measures such as BMI and waist circumference, blood pressure levels, serum glucose and lipid concentrations of the patients and controls were also similar between groups $(p>0.05)$. None of the subjects had renal impairment. The clinical and laboratory parameters of the study group are summarized in the Table 1. Disease duration of the patients was $13 \pm 7.5$ years. BASDAI, BASFI, and BASMI were 3.6 $\pm 2.3,2.9 \pm 2.5$, and $1.9 \pm 2.6$, respectively. Acute phase reactants including hsCRP, and ESR were significantly increased in the patients group $(\mathrm{p}<0.001 ; 8.9 \pm 5.6$ vs. $4.18 \pm 3.71 \mathrm{mg} / \mathrm{L}$, and $11 \pm 19$ vs. $3 \pm 6 \mathrm{~mm} / \mathrm{h}$ respectively). Serum ADMA concentrations were also significantly higher in AS patients than in controls $(1.6 \pm 1$ vs. $0.9 \pm 0.9 \mu \mathrm{mol} / \mathrm{L} ; \mathrm{p}=0.003)$. However, plasma levels of ET-1 were not different between groups $(19 \pm 3.9$ vs. 20.4 $\pm 5.3 \mathrm{pg} / \mathrm{mL} ; \mathrm{p}=0.2$ ).

There were 18 patients $(14 \mathrm{M} / 4 \mathrm{~F} ; 42.3 \pm 10.1$ years $)$ treated with TNF- $\alpha$ blocking agents (9 etanercept, and 9 infliximab). The mean treatment duration for biological drugs was $27.7 \pm 25.8$ months. 30 patients $(22 \mathrm{M} / 8 \mathrm{~F} ; 36.5 \pm 10$ years) were receiving conventional treatments (non-steroidal antiinflammatory drugs and/or sulfasalazine) and none of these subjects had been previously treated with anti-TNF- $\alpha$ agents. Age, sex distribution, BMI, waist circumference, disease duration, acute phase reactants, disease activity indices were similar between anti-TNF- $\alpha$ and conventional treatment groups ( $\mathrm{p}>0.05)$. ADMA and ET-1 concentrations were lower in the anti-TNF- $\alpha$ treated group but these values were not statistically significant $(1.7 \pm 1$ vs. $1.3 \pm 1 \mu \mathrm{mol} / \mathrm{L}$, and $19.3 \pm 4.5$ vs. $18.6 \pm 2.7 \mathrm{pg} / \mathrm{mL}$ respectively). Table 2 summarizes the laboratory and clinical parameters of the groups.

Comparison of three groups (conventional and anti-TNF- 
lar between groups $(\mathrm{p}>0.05)$. Figure 1 represents the dot graphic comparison of ADMA between groups.

Correlation analysis yielded significant correlations between ADMA, hsCRP, LDL cholesterol, HDL cholesterol and triglycerides $(\mathrm{p}<0.05 ; \mathrm{r}=0.2,0.3,-0.2$, and 0.2 , respectively.) On the other hand, disease activity indices, ESR, and ET-1 levels were not correlated with serum ADMA concentrations $(\mathrm{p}>0.05)$.

\section{Discussion}

In this study we demonstrated that serum ADMA levels, reflecting NO metabolism, were increased in AS patients who were free of CV risk factors. It is noteworthy that this finding was obtained in a group of relatively young patients. The other parameter, ET-1, did not differ between patients and controls. To the best of our knowledge, this is the first study investigating ADMA and ET-1 levels in AS patients.

ADMA is an endogenous inhibitor of nitric oxide (NO) and increased levels of this molecule are associated with reduced NO synthesis and vascular dysfunction (20, 21). Elevated ADMA accompanies several CV risk factors including hypertension, dyslipidemia, smoking, insulin resistance and diabetes $(20,21)$. In addition to accompanying the traditional risk factors, it is also recognized as a novel $\mathrm{CV}$ risk parameter, as prospective studies have shown that increased ADMA is associated with a 3- to 5-fold increase in the risk of experiencing serious CV events even in clinically healthy non smoking subjects $(20,22)$. A high ADMA concentration leads to vascular damage by counteracting the physiological effects of NO (such as vasodilatation, inhibition of adhesion molecule expression, inhibition of platelet adhesion, prevention of lipid oxidation), and it thereby promotes atherosclerosis $(20,22)$. In the literature, increased ADMA concentrations have been reported in various inflammatory rheumatic diseases including rheumatoid arthritis (RA) (10), Behcet's disease (12), familial Mediterranean fever (FMF) (11), systemic lupus erythematosus (SLE) (23) and scleroderma (24).
In the present study, there were no subjects with classical $\mathrm{CV}$ risk factors as well as active infection in both groups which might affect the results. On the other hand, it is noteworthy that our ADMA levels were significantly higher in the AS patients compared to controls even in the presence of relatively high hsCRP levels in the control group. In addition, ADMA levels showed a positive and significant correlation with hsCRP. Subgroup analysis of the current study showed that ADMA levels were significantly higher in conventionally treated AS patients than in healthy controls. In contrast, anti-TNF- $\alpha$ treated AS patients had comparable ADMA concentrations compared to healthy subjects, suggesting a beneficial effect of anti-TNF- $\alpha$ treatment on vascular functions in AS. This finding is in accordance with the results of a former study which showed an improvement in endothelial function, by using ultrasonography, after antiTNF- $\alpha$ treatment (25).

Endothelin-1 is a peptide, secreted mostly by vascular endothelial cells, that possesses vasoconstrictor, proinflammatory, mitogenic and proliferative properties (26). Endothelin production is stimulated by mechanical factors such as vascular injury, lipids and cytokines including IL-1 and IL-6 (27). Elevated ET-1 levels have been reported in inflammatory rheumatic diseases particularly in scleroderma (28), SLE (14), Behcet's disease (15) and RA (13). There is no report in the literature with respect to ET-1 levels and AS. In the present study, ET-1 levels were similar between AS patients and controls. As Behcet's disease, scleroderma and SLE may cause direct vascular injury, the presence of higher ET-1 levels in these diseases might not be an unexpected finding.

In conclusion, increased ADMA levels obtained in a group of relatively young AS patients who did not have classical CV risk factors suggests that NO metabolism is impaired in AS. In addition, unchanged ADMA levels in patients treated with TNF blocking agents suggests that antiTNF- $\alpha$ treatment may have a beneficial effect on vascular function in AS.

\section{References}

1. Braun J, Sieper J. Ankylosing spondylitis. Lancet 369: 1379-1390, 2007.

2. van der Linden S, van der Heijde D. Ankylosing spondylitis. Clinical features. Rheum Dis Clin North Am 24: 663-676, 1998.

3. Okan T, Sari I, Akar S, et al. Ventricular diastolic function of ankylosing spondylitis patients by using conventional pulsed wave Doppler, myocardial performance index and tissue Doppler imaging. Echocardiography 25: 47-56, 2008.

4. Kaprove RE, Little AH, Graham DC, Rosen PS. Ankylosing spondylitis: survival in men with and without radiotherapy. Arthritis Rheum 23: 57-61, 1980.

5. Lehtinen K. Mortality and causes of death in 398 patients admitted to hospital with ankylosing spondylitis. Ann Rheum Dis 52: 174-176, 1993.

6. Radford EP, Doll R, Smith PG. Mortality among patients with ankylosing spondylitis not given X-ray therapy. N Engl J Med 297: 572-576, 1977.
7. Smith PG, Doll R. Mortality among patients with ankylosing spondylitis after a single treatment course with $\mathrm{x}$ rays. Br Med $\mathrm{J}$ (Clin Res Ed) 284: 449-460, 1982.

8. Khan MA, Khan MK, Kushner I. Survival among patients with ankylosing spondylitis: a life-table analysis. J Rheumatol 8: 86-90, 1981.

9. Sari I, Okan T, Akar S, et al. Impaired endothelial function in patients with ankylosing spondylitis. Rheumatology (Oxford) 45: 283-286, 2006.

10. Surdacki A, Martens-Lobenhoffer J, Wloch A, et al. Elevated plasma asymmetric dimethyl-L-arginine levels are linked to endothelial progenitor cell depletion and carotid atherosclerosis in rheumatoid arthritis. Arthritis Rheum 56: 809-819, 2007.

11. Terekeci HM, Oktenli C, Ozgurtas $T$, et al. Increased asymmetric dimethylarginine levels in young men with familial Mediterranean fever (FMF): is it early evidence of interaction between inflammation and endothelial dysfunction in FMF? J Rheumatol 35: 2024- 
2029, 2008

12. Sahin M, Arslan C, Naziroglu M, et al. Asymmetric dimethylarginine and nitric oxide levels as signs of endothelial dysfunction in Behcet's disease. Ann Clin Lab Sci 36: 449-454, 2006.

13. Pache M, Schwarz HA, Kaiser HJ, et al. Elevated plasma endothelin-1 levels and vascular dysregulation in patients with rheumatoid arthritis. Med Sci Monit 8: CR616-CR619, 2002.

14. Yoshio T, Masuyama J, Mimori A, Takeda A, Minota S, Kano S. Endothelin-1 release from cultured endothelial cells induced by sera from patients with systemic lupus erythematosus. Ann Rheum Dis 54: 361-365, 1995

15. Ural AU, Yalcin A, Beyan C, Isimer A, Bayhan H. Plasma endothelin-1 concentrations in patients with Behcet's disease. Scand J Rheumatol 23: 322-325, 1994.

16. van der Linden S, Valkenburg HA, Cats A. Evaluation of diagnostic criteria for ankylosing spondylitis. A proposal for modification of the New York criteria. Arthritis Rheum 27: 361-368, 1984.

17. Jenkinson TR, Mallorie PA, Whitelock HC, Kennedy LG, Garrett SL, Calin A. Defining spinal mobility in ankylosing spondylitis (AS). The Bath AS Metrology Index. J Rheumatol 21: 1694-1698, 1994.

18. Calin A, Garrett S, Whitelock H, et al. A new approach to defining functional ability in ankylosing spondylitis: the development of the Bath Ankylosing Spondylitis Functional Index. J Rheumatol 21: 2281-2285, 1994.

19. Garrett $S$, Jenkinson $T$, Kennedy LG, Whitelock H, Gaisford $P$, Calin A. A new approach to defining disease status in ankylosing spondylitis: the Bath Ankylosing Spondylitis Disease Activity Index. J Rheumatol 21: 2286-2291, 1994.

20. Boger RH. Asymmetric dimethylarginine (ADMA): a novel risk marker in cardiovascular medicine and beyond. Ann Med 38: 126136, 2006.

21. Boger RH, Maas R, Schulze F, Schwedhelm E. Elevated levels of asymmetric dimethylarginine (ADMA) as a marker of cardiovascular disease and mortality. Clin Chem Lab Med 43: 1124-1129, 2005.

22. Valkonen VP, Paiva H, Salonen JT, et al. Risk of acute coronary events and serum concentration of asymmetrical dimethylarginine. Lancet 358: 2127-2128, 2001

23. Bultink IE, Teerlink T, Heijst JA, Dijkmans BA, Voskuyl AE. Raised plasma levels of asymmetric dimethylarginine are associated with cardiovascular events, disease activity, and organ damage in patients with systemic lupus erythematosus. Ann Rheum Dis 64: 1362-1365, 2005.

24. Dooley A, Gao B, Bradley N, et al. Abnormal nitric oxide metabolism in systemic sclerosis: increased levels of nitrated proteins and asymmetric dimethylarginine. Rheumatology (Oxford) 45: 676-684, 2006.

25. van Eijk IC, Peters MJ, Serne EH, et al. Microvascular function is impaired in ankylosing spondylitis and improves after $\operatorname{TNF}\{$ alpha $\}$ blockade. Ann Rheum Dis 68: 362-366, 2009.

26. Barton M, Yanagisawa M. Endothelin: 20 years from discovery to therapy. Can J Physiol Pharmacol 86: 485-498, 2008.

27. Goraca A. New views on the role of endothelin (minireview). Endocr Regul 36: 161-167, 2002.

28. Morelli S, Ferri C, Di Francesco L, et al. Plasma endothelin-1 levels in patients with systemic sclerosis: influence of pulmonary or systemic arterial hypertension. Ann Rheum Dis 54: 730-734, 1995.

(C) 2009 The Japanese Society of Internal Medicine http://www.naika.or.jp/imindex.html 